\title{
Cure for a crisis?
}

\author{
Andy-Mark W. H. Thunnissen ${ }^{1,2}$ and Bauke W. Dijkstra ${ }^{1}$
}

\section{The first look at the three-dimensional structure of an essential penicillin binding protein from a human pathogen, and its complex with a $\beta$-lactam antibiotic provides hope for the future design of improved antibiotics.}

'Laboratory of
Biophysical Chemistry
and BIOSON Research
Institute
Department of
Chemistry, University
of Groningen,
Nijenborgh 4, 9747
AG Groningen, the
Netherlands
2Present address:
Melvin Calvin
Laboratory, Structural
Biology Division,
Chemistry
Department,
University of
California at Berkeley,
Berkeley, California
94720, USA

On page 284 of this issue of Nature Structural Biology Pares et al. ${ }^{1}$ describe the X-ray structure of a soluble form of penicillin binding protein $2 \mathrm{x}(\mathrm{PBP} 2 \mathrm{x})$ from the Grampositive bacterium Streptococcus pneumoniae, and its complex with the $\beta$-lactam antibiotic cefuroxime. These are exciting results, that offer a first glimpse of how a $\beta$-lactam antibiotic interacts with an essential target protein and inactivates it with a lethal result to the bacterium. Pharmaceutical industries have been eagerly waiting for such a three-dimensional structure, as knowledge of the precise interactions of the antibiotic with its target enzyme can be used to design antibacterial agents with a higher affinity, or antibiotics that are less prone to induce resistance in the bacterium. The present structure is a significant advance in the ongoing war against the development of antibiotic resistance in pathogenic bacteria.

Penicillin and related $\beta$-lactam antibiotics interfere with the correct processing and metabolism of peptidoglycan, the main structural component of the bacterial cell wall Since no counterpart of peptidoglycan is found in eucaryotic cells, the peptidoglycan metabolism has proven to be an ideal target for these specific antibacterial compounds. Moreover, peptidoglycan synthesis and degradation take place mostly outside the cytoplasm, and therefore the peptidoglycan metabolic enzymes are readily accessible for inhibition by such compounds. Until recently, the use of antibiotics like penicillin has allowed the treatment of almost any bacterial infection $^{2}$. Yet, within the last $20-25$ years resistance mechanisms have evolved in pathogenic bacteria that inactivate the commonly used antibiotics. This so called "crisis of antibiotic resistance" 3,4 has revived research interest in the peptidoglycan metabolic pathways with the objective of characterising existing targets and to identify new ones for the design of novel antibiotics.

\section{Peptidoglycan}

Peptidoglycan (or murein) is a biopolymer, that is built up from two basic elements, linear polysaccharide glycan strands and short oligopeptide chains (see Box). The glycan strands are composed of two alternating sugar residues, $\mathrm{N}$-acetylglucosamine (GlcNAc) and $N$ acetylmuramic acid (MurNAc), covalently connected through $\beta-1,4$ glycosidic bonds. Each of the MurNAc residues carries an oligopeptide side chain bound with an amide bond to its lactyl group. Through cross-links between the oligopeptides of adjacent glycan strands, the peptidoglycan forms a giant network-like structure, that completely encloses the cytoplasmic membrane ${ }^{5}$. The strength and rigidity of the peptidoglycan network allows the bacteria to withstand high osmotic pressures inside their cells and to maintain their specific shapes. Yet, the cell wall should also be flexible to permit growth and division of the organism. These seemingly conflicting requirements of rigidity and dynamic behaviour can be met by the peptidoglycan because of its unique chemical and three-dimensional structure, and an elaborate system of metabolic enzymes to maintain it.

Biosynthesis and assembly of the peptidoglycan have been studied in most detail for Escherichia coli. It is a complex process involving a large number of enzymes (for a review see ref. 6). The building block for the synthesis of the peptidoglycan polymer is the disaccharide-pentapeptide GlcNAc-MurNAcL-Ala-D-
Glu-m-Dap-D-Ala-D-Ala.

This building block is assembled in the cytoplasm of the cell and transported through the cytoplasmic membrane as an undecaprenylpyrophosphate glycoside ${ }^{6,7}$. After arrival in the periplasm the disaccharide-pentapeptide subunits are polymerised into glycan chains and cross-links between adjacent peptide side chains are formed. These reactions are catalysed by membrane-anchored transglycosylases and transpeptidases. The crosslinking reaction always involves the cleavage of the C-terminal D-Ala-DAla peptide bond of the pentapeptide subunits.

\section{Penicillin binding proteins}

The enzymes involved in the crosslinking are the targets of penicillin and related $\beta$-lactam antibiotics, and therefore they are also known as penicillin-binding proteins or PBPs. Penicillins inhibit the cross-linking reaction because of their structural similarity to the geometry of the DAla-D-Ala peptide bond. They form a covalently bound complex with the active-site serine of PBPs that is hydrolysed only very slowly. In $E$. coli nine distinct penicillin-binding proteins have been characterised which are known as PBP1A, 1B, 2, $3,4,5,6,7$ and 8 (for a review see ref. 8). Although the high-molecular weight PBPs (1A, 1B, 2 and 3) have been proposed to be bifunctional enzymes, exhibiting both transglycosylase and transpeptidase activity, the transglycosylase activity has only rigourously been demonstrated for PBP1A and PBP1B. Since the high-molecular weight enzymes have primary roles in the assembly of the peptidoglycan, they are the most lethal targets of the $\beta$-lactam antibiotics.

The lower molecular weight PBPs $(4,5,6,7$ and 8$)$ are involved main- 
offers a wealth of information. As previously deduced from amino acid sequence alignments, the central domain shows a clear similarity to the fold of class $A$ and $C \beta$-lactamases. The active site for the transpeptidase activity is located in this domain, near Ser 337. The bound cefuroxime antibiotic is near two lysine residues, conserved in penicillin binding proteins and class $A$ and $C B$-lactamases. Analysis of the binding mode of cefuroxime will allow to rationalize the interaction of $\mathrm{PBP} 2 \mathrm{x}$ (and other related PBPs) with $\beta$-lactams. The absence of Asp or Glu residues at positions equivalent to the conserved Glu 166 in $\beta$-lactamases, is hypothesized to cause the much slower hydrolysis of the covalent adduct in the PBPs compared to $\beta$ lactamases. The C-terminal domain has no known function; it consists of two small $\alpha \beta \beta \beta$ units. Is its presence indispensable for the viability of the bacterium? The N-terminal domain shows a pair of tongs ready to grip some other molecule: a protein? a piece of peptidoglycan? The precise function of this domain is not known either. Does it have transglycosylase or other activity of its own? Is another protein required to confer catalytic activity to this domain, or is it just needed for the interaction with other components of the cell wall metabolic machinery? These are just a few of the intriguing questions that the new $\mathrm{PBP} 2 \mathrm{x}$ structure raises.

\section{The problem of antibiotic resistance}

In recent years strains of multidrug-resistant pathogens have appeared in hospitals around the world. The infamous methicillin resistant Staphylococcus aureus for MRSA) which may cause potentially fatal hospital infections, is a prime example. To fight the emergence of resistance it is imperative that new or better drugs are developed. Production of $\beta$-lactamases is by far the most frequently encountered way by which bacteria resist $\beta$-lactam antibiotics. $\beta$-Lactamases rapidly hydrolyse the four membered $\beta$-lactam ring of penicillin and related compounds, thus rendering them inactive as antibacterial agents. For this reason there has been a major effort in the past years to generate new $\beta$-lactam antibiotics that are not degraded by $\beta$ lactamases. Cefotaxime and cefuroxime are examples of such $\beta$ lactamase-stable compounds that have been in clinical use for a number of years. Another approach has been to develop powerful $\beta$-lactamase inhibitors, like sulbactam and tazobactam, to protect and potentiate the activity of existing $\beta$-lactams. Although these new compounds have increased the effective treatment of many pathogenic infections, new resistant strains have already emerged that express slightly altered $\beta$-lactamases with an extended substrate-spectrum.

An interesting prospect for $\beta$-lactamase inhibitor design is offered by Strynadka et al. on page 290 of this issue ${ }^{13}$. They have determined at atomic resolution the binding of the $\beta$-lactamase inhibiting protein BLIP to TEM-1 $\beta$-lactamase. BLIP, a 165 amino acid protein produced by Streptomyces clavuligerus, is a highly potent inhibitor of a wide variety of $\beta$-lactamases. It binds to its target by means of a six residue $\beta$ hairpin and interacts with several active site residues that are highly conserved in class A $\beta$-lactamases. It is a fascinating idea to use the conformation of this $\beta$-hairpin as a template for the design of new $\beta$-lactamase inhibitors.

Another way in which bacteria develop resistance is to produce mutants of the target enzymes with a lower affinity for antibiotics: $S$. pneumoniae is a good example of this. Production of $\beta$-lactamases in this organism has never been reported, and its resistance to penicillins and cephalosporins is entirely due to altered penicillin binding proteins. Improved antibiotics that bind with higher affinity, or antibiotics that act on different targets, are needed to fight such pathogens. Although several new antibiotics interfering with bacterial protein synthesis and DNA replication are under development at pharmaceutical companies ${ }^{14}$, the enzymes involved in the metabolism of peptidoglycan are still important targets for antibacterial agents. Almost any step of the known peptidoglycan synthetic reactions can be inhibited by antibacterial sub- stances ${ }^{15}$. Structure determinations of essential enzymes make a rational approach towards the design of better antibiotics feasible. Such efforts have already resulted in the recent $\mathrm{X}$-ray structures of the target of the antibiotic vancomycin ${ }^{16}$, and of MurB, a cytoplasmic enzyme catalyzing the formation of the lactyl ether bond in MurNAc ${ }^{17,18}$.

\section{The future}

The present structure of $\mathrm{PBP} 2 \mathrm{x}$ complexed with cefuroxime offers hope for the rational design of improved antibiotics, even though it has only a limited resolution. The structure has already attracted the interest of industry and it is hoped that analysis of the binding of cefuroxime and other compounds, such as cephalosporins, in the active site will allow the design of antibiotics against $S$. pneumoniae PBP2x with a higher affinity, or a diminished ability to induce resistance without loss of efficacy in treating infections.

Although it remains to be seen whether better antibiotics based on the PBP2x structure can indeed be obtained, the structure will certainly stimulate new ideas for unravelling the role of the enzyme and its homologues in peptidoglycan metabolism. Rationally designed site-directed mutagenesis experiments can now be performed to further characterize the function of PBP2x. The unusual "sugar tong" shape of N-terminal domain suggests that it may be involved in a multi-enzyme complex. Association of peptidoglycan synthases and hydrolases in a multi-enzyme complex could be an attractive possibility for cross-talk between the synthesizing and degrading enzymes. In the Gram-negative $E$. coli bacterium the existence of a multi-enzyme complex has been proposed $^{19}$, which involves the $70,000 M_{\mathrm{r}}$ soluble lytic transglycosylase, and the penicillin-binding proteins PBP3 and PBP7. The structure of this soluble lytic transglycosylase has an intriguing doughnut shaped domain with a large 25-35 $\AA$ central hole ${ }^{20}$, which could very well have a role in the interaction with other components of a peptidoglycan metabolic complex. It is speculated ${ }^{21}$ that the role of 
the transglycosylase would be to attach such a complex to a template strand and, by stepwise cleavage of the strand, move the complex along the strand while the other enzymes take care of the synthesis and inser- tion of adjacent, new peptidoglycan strands. A similar multi-enzyme complex could also take care of the formation and precise cutting of the cell wall septum ${ }^{22}$ that occurs during cell division. The existence of multi- enzyme complexes in Gram-positive bacteria has never been demonstrated: experiments will tell whether PBP2x is part of such a multienzyme complex in the Gram-positive S. pneumoniae.
1. Pares, S., Mouz, N., Pétillot, Y., Hakenbeck, R. \& Dideberg, O. Nature Struct. Biol. 3. 284-289 (1996).

2. Kucers, A. \& Bennett, N.M. in The use of antibiotics: a comprehensive review with clinical emphasis 1-585 (William Heinemann Medical Books, London, 1987)

3. Neu, H.C. Science 257, 1064-1073 (1992)

4. Chin, G.J. \& Marx, J. (eds.) Science 264, 360-393 (1994).

5. Weidel, W. \& Pelzer, H. Adv. Enzymol. 26, 193-232 (1964)

6. van Heijenoort, J. in Bacterial cell wall (eds. Ghuysen, J.-M. \& Hakenbeck, R.) 39-54 (Elsevier Science B.V., Amsterdam, 1994).

7. Holtje, J.-V. \& Schwarz, U. in Molecular Cytology of Escherichia coli 77-119 (Academic Press, London, 1985)

8. Matsuhashi, $M$. in Bacterial cell wall (eds. Ghuysen, J.-M. \& Hakenbeck, R.) 55-71
(Ełsevier Science B.V., Amsterdam, 1994).

9. Jamin, M., Bamblon, C., Millier, S., Hakenbeck, R. \& Frère, J.M. Biochem. I. 292, 735-741 (1993).

10. Kitano, K., Tuomanen, E. \& Tomasz, A. FEMS Microbiol. Lett. 7, 759-765 (1986)

11. Kelly, J.A, et al. J. Biol. Chem. 260, 6449-6458 (1985).

12. Kelly, J.A. \& Kuzin, A.P. J. Mol. Biol. 254, 223-236 (1995)

13. Strynadka, N.L. et al. Nature Struct. Biol. 3 290-297 (1996)

14. Service, R.F. Science 270, 724-727 (1995).

15. Gale, E.F.E., Cundliffe, E., Reynolds, P.E., Richmond, M.H. \& Waring, M.J. in The molecular basis of antibiotic action 49-120 (John Wiley \& Sons, New York, 1972).

16. Fan, C., Moews, P.C., Walsh, C.T. \& Knox, J.R. Science 266, 439-443 (1994)

17. Benson, T.E., Filman, D.J., Walsh, C.T. \& Hogle,
J.M. Nature Struct. Biol. 2, 644-653 (1995)

Benson, T.E. Walsh, C.T. \& Hogle, J.M Structure 4, 47-54 (1996).

19. Romeis, T. \& Höltie, J.V. Eur J. Biochemi 224, $597-604$ (1994)

20. Thunnissen, A.M.W.H. et al. Nature 367 750-753 (1994)

21. Thunnissen, A.-M.W.H. PhD. Thesis, Groningen Universtiy (1995)

2. Wienties, F \& Nanninga, N. J.Bacteriol 171 3412-3419 (1989).

23. Schleifer, K.H. Meths Enzymol. 18, 123-156 (1985).

4. Labischinski, H. \& Maidhof, H. in Bacterial cell wall (eds. Ghuysen, J.-M. \& Hakenbeck, R.) 23-38 (Elsevier Science B.V., Amsterdam, 1994)

25. Glauner, B., Höltje, J.-V. \& Schwarz, U. J. Bio Chem. 263, 10088-10095(1988).

26. Garcia-Bustos, J.F Chait, B.T \& Tomasz A. J. Biol. Chem. 262, 15400-15405 (1987).

\section{Bundled up against the heat}

Histones are the first-line managers of the cellular structure of all eucaryotic chromosomes, organizing DNA into nucleosomes. In general, procaryotes must rely on other means, perhaps as

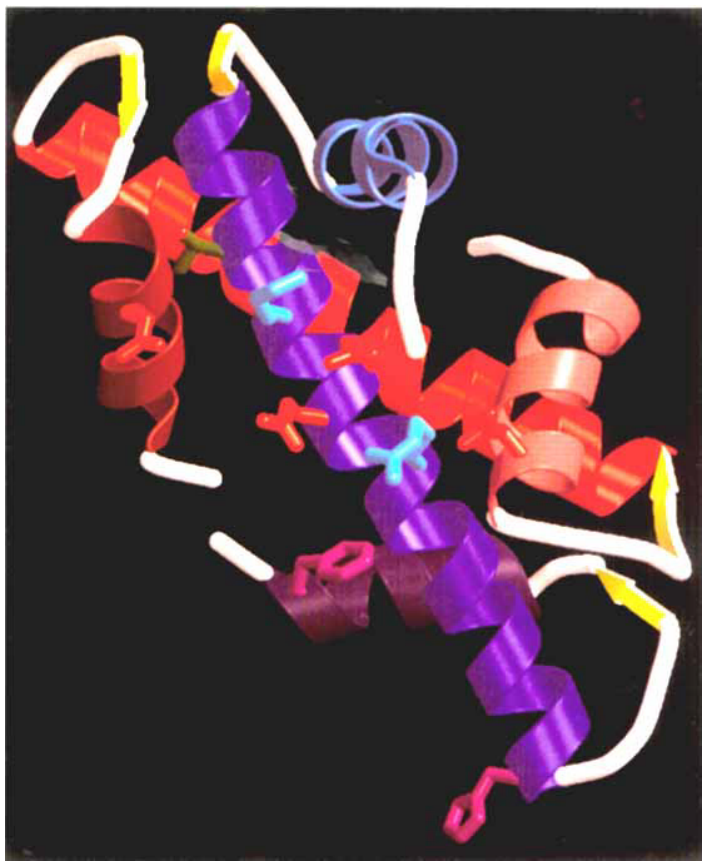
befits the relative simplicity of their genomes. However, one division of the procaryotes, the archaea, do have histones, based on the homology of sequence, function, and now structure-as reported by Mary R Starich, Kathleen Sandman, John N Reeve and Michael F Summers in J. Mol. Biol. 255, 187-203 (1996) and shown in the picture-lending further credence to the idea that eucaryotes descended from archea.

The NMR structure of the histone HMfB, from Methanothermus fervidus, confirms that it functions as a dimer (one monomer shown with red helices, the other with blue). An individual monomer possesses the classical 'histone fold' seen in more prosaic organisms, like chickens. The solution dimer resembles the $\mathrm{H} 2 \mathrm{~A}-\mathrm{H} 2 \mathrm{~B}$ or $\mathrm{H} 3-\mathrm{H} 4$ dimers in eucaryotes.

$M$. fervidus is an extreme thermophile, and living in temperatures in excess of $80^{\circ} \mathrm{C}$ presents it with special challenges. Strategically positioned residues (side chains shown in the picture) may lend stability to the dimer through hydrogen bonds and salt bridges. For example, an electrostatic interaction between Arg 37 in the long helix (blue) of one monomer and Asp 14 in helix 1 of the other (pink) may help keep the $C$ termini tacked down. 